\title{
Food Safety and Quality Data Management Using Artificial Intelligence
}

\author{
Mehrdad Tajkarimi, DVM MPVM PhD \\ EAS Independent Consultant, LLC 1700 Diagonal Road, Suite 750, Alexandria, VA 22314, USA
}

\section{SUMMARY}

Are you overwhelmed with operational food safety and quality data and uncertain how to organize and analyze it to provide justification that your food safety and quality program is effective? Then you have a lot of company. The food industry's access to data is not the limiting factor it may have been 20 years ago. The limiting factor today is having more data than there is capacity to analyze and process into meaningful trends. Is there a solution that is available and practical?

\section{OVERVIEW}

The food industry is in need of a holistic approach to organize, analyze, integrate, and generate conclusions that can help benchmark and track key performance indicators (KPIs) related to food safety and quality (12). The application of machine learning and artificial intelligence (AI) can provide the conceptual tool to transform food safety and quality data management, driving it from a parallel and repetitive control base model toward a value-based food safety and quality system (13). In short, AI can deliver on the need to have a feedback loop for existing food safety and quality programs and whether they are meeting the needs and expectations of company quality assurance management and generate frequent analytical information that can be summarized in reports to company senior management teams (8). Other outcomes of using AI in this manner are to provide a number-based system to justify investment in a company's food safety and quality programs, redirect resources to where they have the most impact, and potentially lower food safety and quality costs while enhancing delivery of superior food safety and quality control and management (15).

\section{Section I - What are AI and algorithms?}

$\mathrm{AI}$ is an area of computer science that emphasizes the creation of intelligent machines that work and react similarly to humans. Industries that were early adopters of AI include speech recognition, facial recognition, biometrics, planning, and computer-based problem solving. AI algorithms learn and improve their effectiveness similar to the learning process for human food safety and quality professionals (7). In the AI learning process, structured data are fed into the computer systems and identified with a label or annotation to be recognizable to the algorithm's data point (13). The algorithm starts to examine the input data and compare it with known data that the algorithm already has analyzed. The results can then be modified by receiving more data inputs or can solve equations for the human operator. The algorithms can learn from data-either numerical, such as colony count or $\mathrm{pH}$, or a statement, such as auditor note (1) — creating an output such as a simple grouping of data with means, modes, etc., or more valuable outputs such as statistical probability or a classification or categorization. The more data fed into the algorithm, the more learning, data outputs, probabilities, and classifications (Fig. 1). A real-life example is the use of weather data placed into AI-based algorithms (models) to provide short- and long-range weather forecasts $(6,7)$.

AI's reach and applicability are expanding rapidly. In the health care industry alone, investments in this technology are predicted to reach $\$ 6$ billion by 2021 (6). What does AI mean for the food processing industry, and how can it be used to benefit food safety?

$\mathrm{AI}$ is enabling computers to think and learn in a manner to similar humans. Google and Facebook are already using it (e.g., search suggestions and photo recognition). The use of AI as a tool for food safety and quality is in its infancy. However, with effort and investment, it has the potential to emerge as a game changer, forming a structural foundation to incorporate facility, industry, and government data to form a complete picture of risks, vulnerabilities, and opportunities for improvement (Fig. 2) (6).

\section{Application and example of AI for food safety and quality analysis \\ One successful application of AI is with the Chicago Department of Public Health $(4,5)$. The department analyzes vast amounts of data on previous violations related to food safety, including time since the last inspection, operation period, nearby garbage and sanitation complaints, 3-day high-temperature readings, nearby burglaries, and tobacco and alcohol licenses issued by Chicago Department of Public Health to help identity at-risk restaurants. Collectively, these form a picture of the potential for food safety or quality risks.}




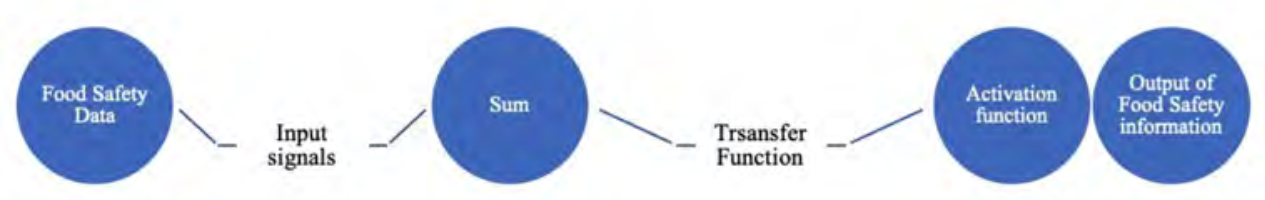

FIGURE 1. Artificial intelligence process of data.

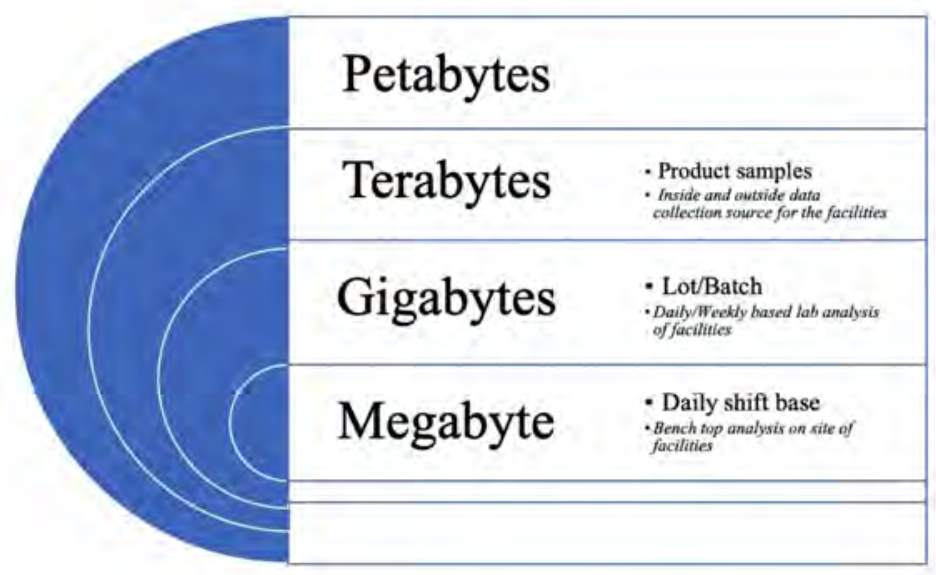

FIGURE 2. Food Safety big-data base structure for developing AI.

In this system, the processing is conducted in Pythonbased software. The algorithm output is to designate high- and low-risk restaurant and public places using the AI system and following the indicated KPIs designates by the food safety expert for the AI system. The AI results and the software design are publicly available at the Food Protection Division-Food Inspection Reporting System for the City of Chicago and in GitHub $(4,5)$.

For an AI solution to be successful, it requires the input of a significant amount of data to allow the algorithm to learn and optimize its performance.

\section{Section II - Food safety and quality data structure}

It is valuable at this stage to discuss the current structure and condition of food safety and quality data, regardless of whether AI is used. There are two categories for food safety and quality data: internal and external. External data such as product recalls, foodborne outbreaks, relevant electronic health records, and finished product testing help to form a more complete picture and are being actively collected by numerous nongovernmental and governmental organizations.

An example of food safety data analysis in action is the Foodborne Diseases Active Surveillance Network (2), managed by the Centers for Disease Control and
Prevention (CDC) in collaboration with 10 emerging infections program (EIP) sites. EIPs are population-based networks of the CDC and state health departments, correlated with local health departments, public health laboratories, clinical laboratories, infection control practitioners, health care providers, academic institutions, and other federal agencies. EIPs use these sources of raw data and then organize, combined, monitor, interpret, and evaluate to provide emerging infectious disease tracking, identification, prevention, and control approaches (2). Other government-based examples include the U.S. Department of Agriculture, which maintains a useful database about cost estimates of foodborne illnesses (15), and the U.S. Food and Drug Administration, which collects data from the Reportable Food Registry, food recalls, and warning letters and maintains positive lists of allowable food additives (generally recognized as safe database), food contact substances, etc.

Internal data include sanitation verifications, pest control programs, internal audits, supplier verification, consumer complaints, hazard analysis critical control point and preventive control data, good manufacturing practice and supplier verification data, foodborne illnesses, and company-generated internal and external lab records (12). 
Human foodborne illnesses and diseases are considered in both internal and external AI data analysis. Examples include the following:

i. The most commonly reported foodborne diseases are listeriosis, salmonellosis, campylobacteriosis, and illness triggered by Shiga toxin-producing strains of Escherichia coli (15).

ii. Other zoonotic (transmitted by animal) foodborne diseases such as brucellosis are a significant public health issue in developing countries $(9,16)$.

iii. Trichinellosis and echinococcosis are diseases caused by animal parasites in humans.

iv. Antimicrobial resistance, caused by increasing usage of antibiotics in animal feed, is considered.

v. Persistent organic pollutants, acrylamide, pesticides, and dioxin represent a public health risk; all are chemical contaminants and hazards in food (16).

What is the best approach for the food industry to collect, organize, and analyze its food safety data and make a difference? There are three key elements for data collection in food safety:

i. Food safety and quality KPIs and monitoring. Determine all possible indicators that might affect food safety and quality and determine the best approaches to monitor those parameters and variables. Food safety and quality data in a manufacturing plant can be collected using automated sensors that feed data to computers, as well as by individuals. Examples include ATP readings, $\mathrm{pH}$, temperature, and composition of inprocess product; metal detectors; and optical scanners. Outside contract laboratory testing can provide data on $\mathrm{pH}$, microbial load (environmental and possibly finished-product pathogen testing), allergen residues, and labeling compliance, which are also important sources of food safety data.

ii. Design of experiments and/or predictive modeling using AI and machine learning approaches. These systematic approaches optimize the impact of input variables from food safety and quality data measurement with the results of the food safety system implementations (outcomes). Several available software applications used to conduct these types of experimental designs incorporate practical and easy-tounderstand approaches $(10,11)$. Programs such as the Pathogen Modeling Program and ComBase $(3,14)$ are examples of predictive modeling that are familiar to the food processing industry.

iii. Action on the completed information. Conversion of the data into actionable information is the most important part of the food safety and quality database implementation system. The overall structure of the food safety and quality database incorporates all elements identified in Fig. 3 to form a holistic picture.

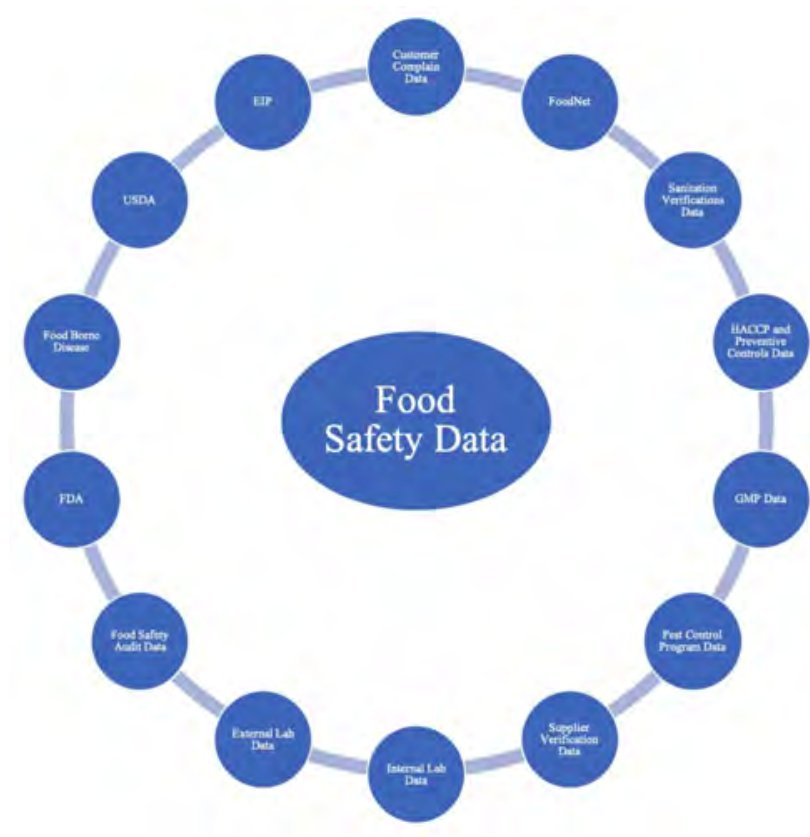

FIGURE 3. Elements of Food Safety and Quality “Big Data.”

\section{Challenges of using AI in food safety and quality programs}

Food safety data have tremendous diversity in format, type, and context. Therefore, merging big food safety and quality data into conventional databases is challenging and hard to implement.

One of the challenges for food safety professionals is that the people creating algorithms for food safety and quality purposes are not the food safety professionals; therefore, both food safety professionals and computer specialists need to learn more about each other's profession or employ a translator that understands the interests and professions of both. Food safety professionals and programmers will need to work together.

\section{Other challenges face food safety using AI:}

i. Industrial data release. Accessing confidential industrial food safety and quality records is strictly protected. Issues such as data ownership and the right to access or sell data to other AI and non-AI companies include potential security breach consequences related to the food safety data.

ii. Data interpretation. Internal and external inspector and auditor notes are unstructured, making them difficult to interpret and process. Although all data are valuable, the variability and inconsistencies in the format and location of the data require additional effort to organize the data into a standard structure, which wastes resources and drives up the operations costs to use the AI technology effectively. 
iii. Data inaccuracy. All data must undergo some type of clean or verification testing to ensure they are accurate and representative of what they are measuring.

iv. Regulatory requirements. Relatively new food safety laws and regulations in many countries allow government inspectors to have access to all food safety and quality data, including that generated as the result of using AI.

v. Liability. The liability of the AI processed data is important. Does the use of AI technology create a larger liability burden for a company related to its food safety and quality programs? Similarly, what is the impact related to liability insurance companies agreeing to insure food manufacturing companies using $\mathrm{AI}$ as a tool?

vi. Trust. Is the food manufacturer's senior management willing to trust a food safety and quality-related prediction from a software algorithm instead of a human?

\section{The future outlook for AI for food safety and quality}

Hybrid models of AI application can be the best tools for improving food safety and quality programs for food manufacturers by using internal and external data points collected from many sources and then integrating and analyzing them to predict the likelihood of unfavorable food safety events. The application of AI with human instinct and experience from internal and external inspectors, auditors, and food safety and quality professionals will support detection, preventive action, and identification of risk factors, recognizing that food safety and quality professionals and company senior management retain the ultimate responsibility for making the right food safety and quality decisions.

\section{CONCLUSION}

With the government and food industry focus on prevention of foodborne illness, having predictive tools that can identify when the food safety and quality risk has crossed the acceptable threshold is a necessity $(6,15)$. Food safety is considered a high-risk operation by the U.S. Government Accountability Office, the investigative arm of the U.S.

Congress (12).

The industry is taking small steps toward addressing key issues using AI-powered solutions, but there is a long way to go before this tool becomes a routine part of a food manufacturer's food safety and quality program. AI technology is available today and has proved its value in other industries, such as medicine, so the time to use it in the food safety and quality arena is now.

\section{REFERENCES}

1. Allan, T., and R. Piston. 2017. Manage food safety data. Food Safety Strategies. Available at: https://www.foodsafetystrategies.com/ articles/469-manage-food-safety-data.

2. Centers for Disease Control and Prevention. 2019. Foodborne disease active surveillance network. Available at: https://www.cdc.gov/ foodnet/index.html.

3. ComBase. 2020. ComBase tools. Available at: https://www.combase.cc/index.php/en/8category-en-gb/21-tools.

4. Food Inspection Reporting System. 2018. Chicago food inspection. Available at: https://github.com/trupti-jadhav/FoodInspection-in-City-of-Chicago-Python/blob/ master/FOOD_INSPECTION_FINAL. ipynb.

5. Food Inspection Reporting System. 2020. City of Chicago health inspection service. Available at: https://webapps1.chicago.gov/ healthinspection/inspection.jsp.

6. Gin, A., and B. Helwig. 2018. FDA signals fast-track approval for AI-based medical devices. Bloomberg Law. Available at: https://news.bloomberglaw.com/tech-andtelecom-law/fda-signals-fast-track-approvalfor-ai-based-medical-devices-1.
7. Greenfield, D. 2019. Artificial intelligence in medicine, applications, implications, and limitations. Harvard University blog. Available at: http://sitn.hms.harvard. edu/flash/ 2019/artificial-intelligence-inmedicine-applications-implications-andlimitations/.

8. Hatch, D. 2019. Do you know what your food safety analytics are telling you? Corvium blog. Available at: https://www.corvium. com/blog/do-you-know-what-your-foodsafety-analytics-are-telling-you.

9. Heredia, N., and S. Garcia. 2018. Animals as sources of foodborne pathogens: a review. Anim. Nutri. 4(3):250-255. Available at: https://www.ncbi.nlm.nih.gov/ pmc/ articles/PMC6116329/.

10. MoreStream. 2020. Design of experiment. Available at: https://www.moresteam.com/ toolbox/design-of-experiments.cfm.

11. QI Macros. 2020. Design of experiment for Excel. Available at: https://www.qimacros. com/lean-six-sigma-articles/doe-software/ ?gclid=Cj0KCQjw2PP1BRCiARIsAEqv-pR QCmd80a0PW10nVt6OeabANx7o0Xnwd2 Of-Yp5cIpicGF3BR2x9ZcaAmJoEALw_wcB.
12. Rumpf, A. 2007. Leveraging food safety data to improve operations. Food Safety Magazine. Available at: https://www. foodsafetymagazine.com/magazine-archive1/ augustseptember-2007/leveraging-foodsafety-data-to-improve-operations/.

13. Marshall, S. 2020. How AI is transforming the future of healthcare. International SOS. Available at: https://www.internationalsos. com/client-magazines/in-this-issue-3/howai-is-transforming-the-future-of-healthcare.

14. U.S. Department of Agriculture. 2020. Pathogen modeling program (PMP) online. Available at: https://pmp.errc.ars.usda.gov/ PMPOnline.aspx\#nogo.

15. U.S. Department of Agriculture Economic Research Service. 2014. Cost estimates of foodborne illnesses. Available at: https:// www.ers.usda.gov/data-products/costestimates-of-foodborne-illnesses/costestimates-of-foodborne-illnesses/\#Pathogen.

16. World Health Organization. 2020. Foodborne illness data and statistics. Available at: http://www.euro.who.int/en/ health-topics/disease-prevention/foodsafety/data-and-statistics. 